\title{
The effect of cohesion on the discharge of a granular material through the orifice of a silo
}

\author{
Adrien Gans ${ }^{1 *}$, Pascale Aussillous ${ }^{1}$, Blanche Dalloz ${ }^{1}$, and Maxime Nicolas ${ }^{1}$ \\ ${ }^{1}$ Aix Marseille Univ, CNRS, IUSTI, Marseille, France
}

\begin{abstract}
We present the results of both experimental and numerical investigations of the silo discharge for a cohesive granular material. In our study, thanks to a cohesion-controlled granular material (CCGM) we propose to investigate the effect of the cohesive length $l_{c}$, on the discharge of a silo for two different configurations, one axisymmetrical, and one quasi-2D rectangular silo. In both configurations, an adjustable bottom is used to control the size of the orifice. As observed for cohesionless granular material by previous studies, the mass flow rate and the density through an orifice are mostly controlled by the diameter of the orifice $D$. The experimental results of the quasi-2D silo are compared with continuum numerical simulations.
\end{abstract}

\section{Introduction}

Cohesive granular materials are encountered in many geophysical and industrial applications, examples being fine sand, cement, pharmaceutical powders and flours $[1,2]$. Whereas many advances have been made in the description of cohesionless granular flows in various configurations, the behavior of cohesive powders remains elusive. In particular, one difficulty lies in the understanding of the cohesion force between the particles. For very small particles (typically below $10 \mu \mathrm{m}$ in diameter), attractive forces like Van der Walls [3] or electrostatic forces [4] are dominant whereas for larger particles, humidity brings the necessary amount of water to build strong liquid bridges between particles and therefore creates a bulk cohesion [5]. In industrial or geophysical situations, the cohesion force between grains may evolve due to the variation of the environment (confinement pressure, change of temperature, change of humidity rate) or due to the micro-mechanical evolution of the bonds (ageing, sintering, chemical reaction). Since the concept of cohesion overlaps a great complexity, there is a need of a simple definition and a good control of the cohesion to perform quantitative experiments. Several experimental studies have already been conducted using wet granular materials [6] where the cohesion arises from capillary bonds, but the main difficulty lies in the ability to control and reproduce the cohesion force between particles. We recently developed a cohesion-controlled granular material (CCGM) [7] where the cohesion originates from a polymer coating of glass particles. This CCGM has proven to be very useful to develop experimental studies where a bulk cohesion needs to be implemented. Among the possible experimental configurations, an inclined plane setup and a granular collapse configuration have already been investigated. Using this

*e-mail: adrien.gans@univ-amu.fr recently developed method to create a cohesion-controlled granular material, we experimentally consider the mass flow rate of cohesive spherical grains through the orifice of a silo. This configuration has been extensively studied in the case of dry granular material. Several studies already showed that the mass flow rate through an orifice is mostly controlled by the diameter of the orifice $D$, and, on a second order, the diameter of the grains $d$ [8]. More recently, investigations on the local dynamics have shown that the velocity and the density near the orifice follows a self-similar profile where the value at the center of the orifice is governed by $D$ and $d$ [9-11]. Other studies focused on the jamming conditions during silo flows $[12,13]$. In this study, we characterize the effects of the cohesion on the initiation of a flow, as well as the effect on the mass flow rate of a cohesive granular material through the circular orifice of an axisymmetric silo. We also investigate the effect of cohesion on the velocity profile at the orifice of a quasi-2D silo. Our experimental results for the quasi2D configuration are compared to numerical simulations based on a Navier-Stokes solver (Basilisk $[14,15]$ ) with a cohesive $\mu(I)$ granular rheology performed with the help of A. Abramian and P.-Y. Lagrée at d'Alembert Institute, Paris, in the framework of the COPRINT project (ANR17-CE08-0017). This solver has already given interesting results for the silo configuration [14] and the collapse of cohesive granular media [15].

\section{Experimental and numerical methods}

In this section we present the methods we used to process our experiments and simulations on different configurations.

In the experiments, the controlled-cohesion granular material (CCGM) consists of glass beads of radius $d=$ $340 \mu \mathrm{m}$ and $d=800 \mu \mathrm{m}$, coated with polyborosyloxane 
(PBS) made from a-OH terminated Polydimethylsiloxane (PDMS) cross-linked with boric acid $\left(\mathrm{H}_{3} \mathrm{BO}_{3}\right)$ [7]. The thickness $b$ of the PBS layer coating the glass beads is the control parameter of the inter-particle cohesion. The inter-particle force is captured by the expression $F_{c}=$ $1.5 \pi \gamma d\left(1-\mathrm{e}^{-b / B}\right)$ where $B \approx 230 \mathrm{~nm}$ is a characteristic length and $\gamma=24 \mathrm{mN} \cdot \mathrm{m}^{-1}$ is the surface tension of the PDMS [7]. The cohesive stress of the bulk material $\tau_{c}$ is linearly related to the microscopic inter-particle force $F_{c}$, therefore changing the coating thickness $b$ allows to control the bulk cohesion of the granular material. To describe the flow under gravity, we define the cohesion length $l_{c}=\tau_{c} /(\phi \rho g)$, which represents the characteristic length where gravity effects are balanced by cohesion effects, with $\rho$ the bulk density and $g$ the free-fall acceleration. The experiments are performed using several batches of particles from cohesionless granular beads to cohesive grains with $l_{c}=2.6 \mathrm{~mm}$.

\subsection{Silo Experiments}

The set-ups of the axisymmetrical and the quasi-2D silo are presented in fig 1 . The axisymmetric silo is $60 \mathrm{~mm}$ wide for $50 \mathrm{~cm}$ high. The quasi-2D silo is $11 \mathrm{~cm}$ wide and $60 \mathrm{~cm}$ high with a thickness of $2 \mathrm{~cm}$. For both silos, the removable bottom is used to change the size of the orifice from $1 \mathrm{~mm}$ to $30 \mathrm{~mm}$. At the beginning of the experiment, the height of the column of grain, and the mass of grains poured in the silo gives us the initial volume fraction $\phi_{b}$ of the material. When the orifice is opened, the mass flow rate is recorded with a weighing scale with a $20 \mathrm{~Hz}$ frequency. We first investigate whether the cohesion is completely preventing the initiation of the flow depending on the diameter of the orifice, then we investigate the effect of the cohesion on the mass flow rate. We also investigate the velocity profile near the orifice of the quasi-2D silo. These velocity profiles are obtained by recording the flow at the orifice with a high-speed camera and processed by PIV algorithm to access to the velocity field close to the orifice.

(a)
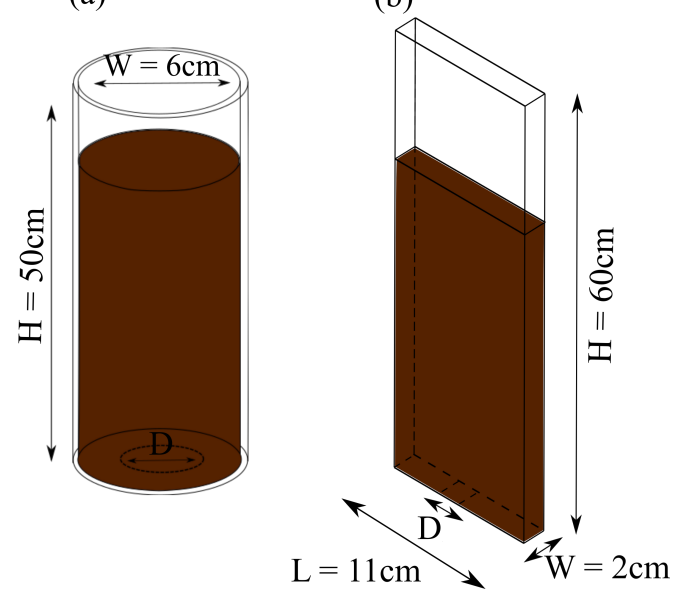

Figure 1. Geometry of the axisymmetric silo (left) and the 2D silo (right).

\subsection{Numerical simulations}

The experimental measurements of the quasi-2D silo are compared to a numerical model based on a 2D NavierStokes solver (Basilisk [14, 15]) including a simple cohesive granular rheology. Without cohesion, the granular rheology is modelled with the now classical $\mu(I)$ constitutive law, where the friction coefficient is a function of the dimensionless inertial number $I$.

$$
\mu(I)=\mu_{s}+\frac{\Delta \mu}{I_{0} / I+1}, \quad I=\frac{\dot{\gamma} d}{\sqrt{P / \rho}} .
$$

Where $\mu_{s}$ is the friction coefficient of the granular material and $P$ is the pressure. In our model, this rheology is enhanced with the cohesion between particles which is represented as a yield stress $\tau_{c}$ so that the tangential stress $\tau$ is

$$
\tau=\tau_{c}+\mu(I) P .
$$

The cohesive property of the material is here depicted by a yield stress $\tau_{c}$ that has to be overcomed to trigger the flow. In the numerical model, we use the width of the silo $L_{\text {num }}$ as the scaling parameter for normalisation. The numerical cohesive length is defined $l_{\text {num }}=\tau_{c} / \rho g L_{\text {num }}$. This dimensionless cohesive length is used as the parameter to control the cohesion in the simulations.

\section{Results}

\subsection{Axisymmetric silo}

We first investigate the threshold of flowability for the axisymmetric silo. The results are presented in fig 2 . For cohesionless grains, a threshold is seen around $D=4 d$ which is a known result from a previous studies [13]. For a cohesive length $l_{c} / d<1$, the threshold is delimited by the diameter of the grains. For $l_{c} / d>1$, the threshold is given by $D=4 l_{c}$. It appears that for cohesive granular material, the cohesive length is replacing the size of the grains for the threshold of flowability.

Considering that result, we suggest to introduce a simple parameter $d^{*}=\max \left[d, l_{c}\right]$ to take in count the fact that the cohesive length acts like an effective grain size when $l_{c}>d$. The threshold of flowability is now given by $D=4 d^{*}$

When the flow occurs, for orifices larger than four times the cohesion length, we investigate the effect of the cohesive length on the mass flow rate. From previous study [10], we can express the mass flow rate as a function of the size of the beads, and the diameter of the orifice.

$$
Q=C \rho_{p} \phi_{b} \sqrt{g D^{5}}\left[1-\alpha e^{\frac{\beta D}{d}}\right] .
$$

Where $\phi_{b}$ is the bulk volume fraction, $\rho_{p}$ is the density of the beads , $\mathrm{C}, \alpha$ and $\beta$ are fit parameters.

The trend $Q \propto \sqrt{g D^{5}}$ is observed in fig 3, even for strong cohesion, and the increase of cohesion is correlated to a decrease of mass flow rate. This shows that the diameter of the orifice is the main parameter to control the mass flow rate and cohesion has only a second order effect comparable to the effect of the grain size. 


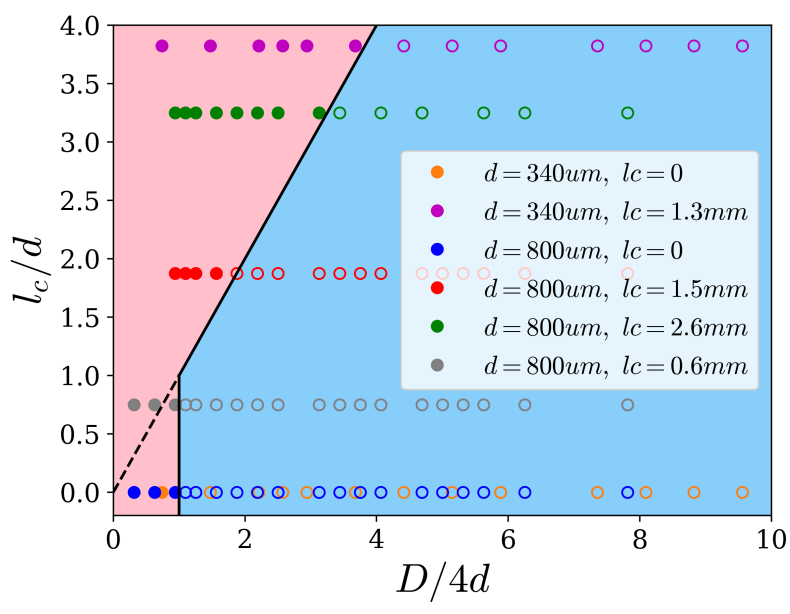

Figure 2. Threshold of flowability depending on the cohesion $l_{c}$, the size of the grains $d$ and the diameter of the orifice $D$. The pink region is the non-flowing zone, and the blue region is the flowing zone. Empty symbols correspond to flowing experiments, and full symbols correspond to non flowing experiments.

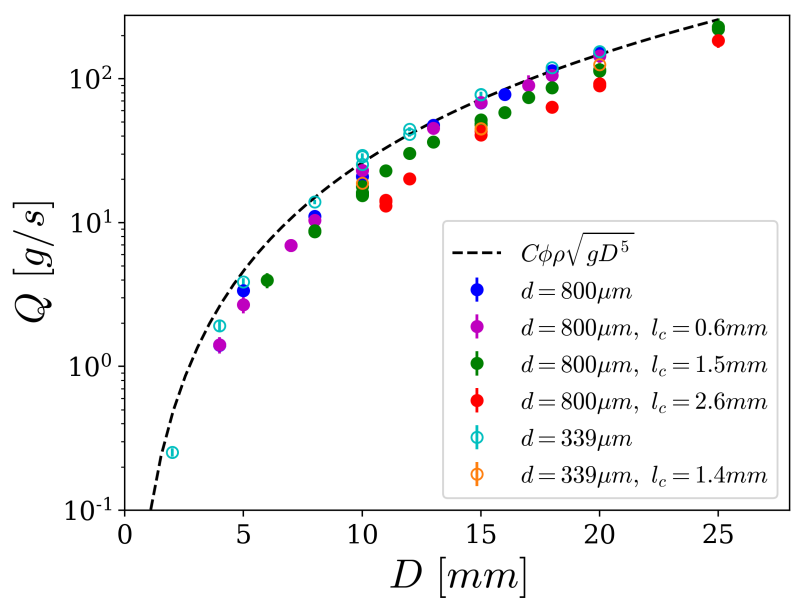

Figure 3. Mass flow rate as a function of the orifice diameter for several cohesions for an axisymmetric silo.

We propose slightly to modify the expression of 3 by using $d^{*}=\max \left[d, l_{c}\right]$ instead of $d$. The results compared to the theoretical prediction are shown in fig 4 . As we see, all the data collapse on a single master curve and the cohesive length seems to act like an effective particle size for $l_{c}>d$.

\subsection{Quasi-2D silo}

In this section, we investigate the velocity profile at the orifice of a quasi-2D silo. The PIV allows us to have access to the velocity field close to the orifice while the weighting scale records the mass flow rate. Qualitatively, the first effect of cohesion is the obvious effect on the morphology of the flow. As observed in fig 5 (a), the flow of cohesive grains is very narrow which is unusual for cohesionless grains. Surprisingly, although the velocity field seems to depends on cohesion, the auto-similarity of the velocity

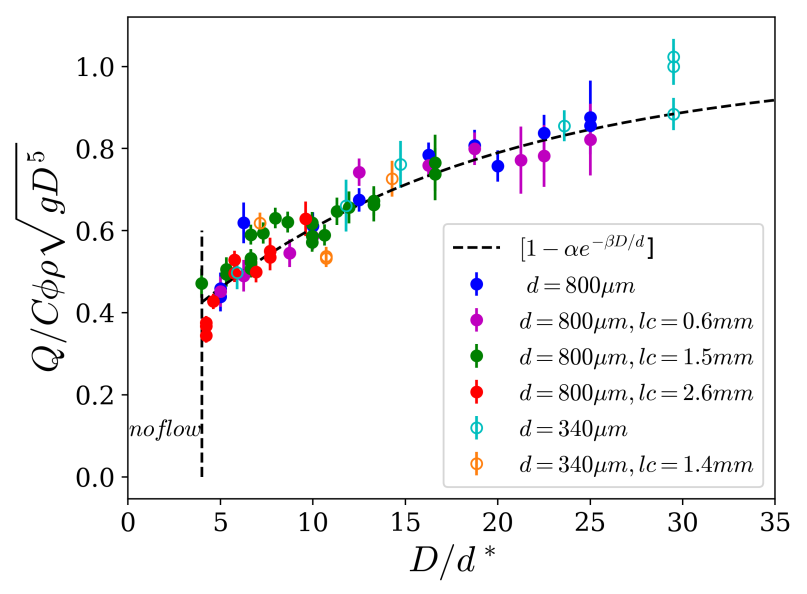

Figure 4. Rescaled mass flow rate. $C=0.62, \alpha=0.74, \beta=$ 0.063

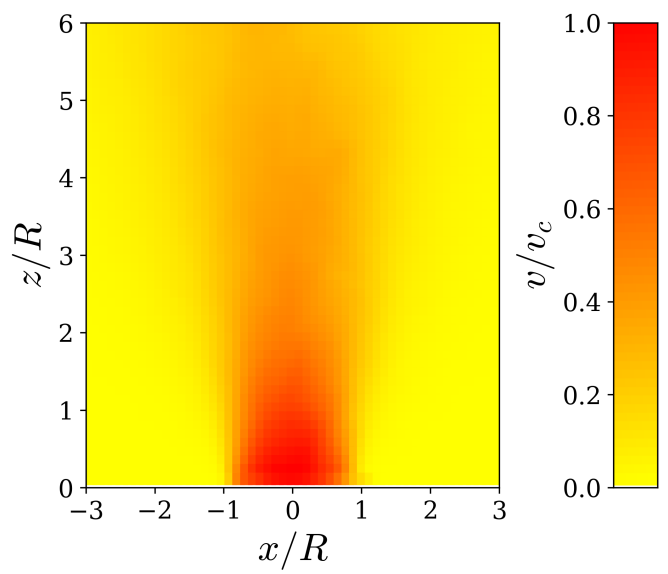

(a)

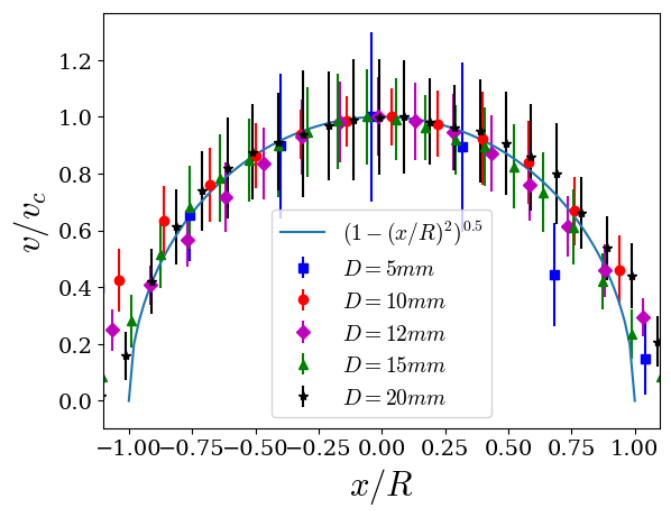

(b)

Figure 5. (a) Normalised velocity field for $D=20 \mathrm{~mm}$ for cohesive grains, $l_{c}=2.6 \mathrm{~mm}$. (b) Normalised velocity profile at the orifice. $v_{c}$ is the velocity at the center of the orifice.

profile close to the orifice does not seem to be affected by cohesion. This result is consistent with the results of section 3.1: the mass flow rate seems mostly governed by the diameter of the orifice. 


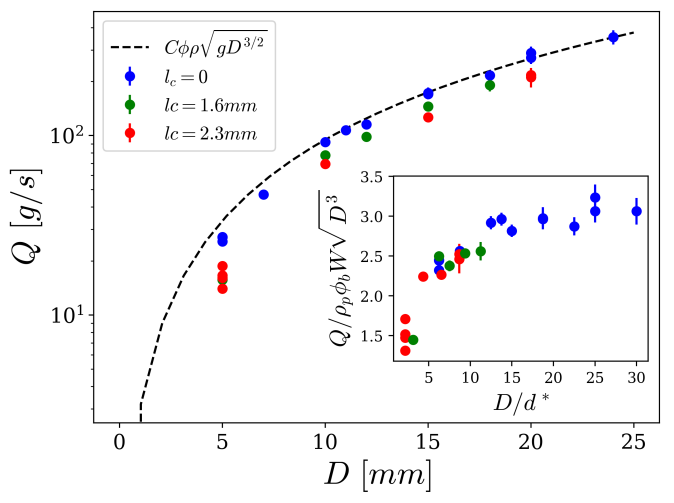

(a)

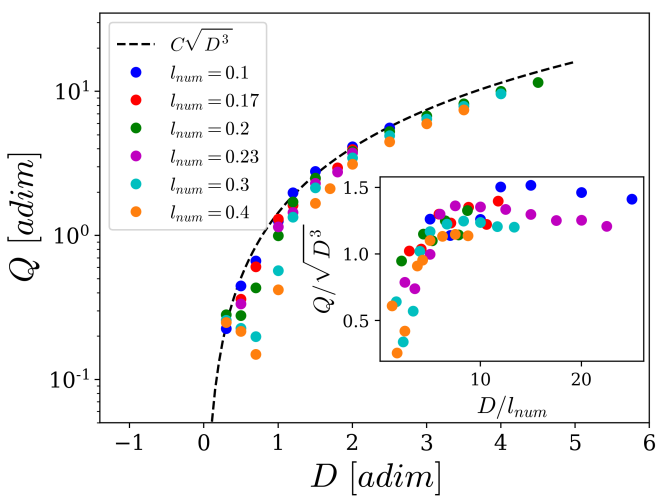

(b)

Figure 6. (a) Mass flow rate as a function of $D$ for several cohesion. Inset : Normalised mass flow rate as a function of $D / d^{*}$. (b) Numerical mass flow rate as a function of $D$. Inset : normalised numerical mass flow rate as a function of $D / l_{c}$.

This result is also consistent with the measurements of the mass flow rate with the weighting scale. For a quasi$2 \mathrm{D}$ silo, the mass flow rate is given by the equation :

$$
Q=C \rho_{p} \phi_{b} W \sqrt{g D^{3}}\left[1-\alpha e^{\frac{\beta D}{d}}\right] .
$$

Where $\mathrm{W}$ is the width of the quasi-2D silo. A similar behavior as the axisymmetric silo is observed on fig 6(a). The trend $Q \propto W^{3 / 2}$ is observed, and this trend is also captured by the numerical model on fig 6(b). Similarly as the axisymmetric, we can rescale every data on a single master curve by using the parameter $d^{*}$. This scaling is less convincing for numerical simulations, however the numerical model does not consider the volume fraction of the granular material.

The global trend that can be observed in figs 6 (a) and (b) is quite similar. It tends to confirm that the global behavior of the dynamics is captured by the numerical model, even if the numerical model does not include the volume fraction. Further results about the comparison be- tween experiments and numerical simulations will be presented at the conference.

\section{Conclusion}

Using a cohesion-controlled granular material, we investigated several configurations of the discharge of a silo. We observed a modification of the scaling laws when the cohesive length is higher than the diameter of the grains, and we reported that there is a threshold of flowability governed by a new parameter $d^{*}=\max \left(d, l_{c}\right)$. Our results suggests that the cohesive length is acting like an effective grain diameter when the cohesion becomes sufficiently strong. The similar trend observed in the results of both numerical simulations and quasi-2D experiments shows that a yield stress added to a $\mu(I)$ rheology seems relevant. These experiments provide an improved understanding of the behavior of flowing cohesive granular materials.

\section{References}

[1] A. Cannavacciuolo, D. Barletta, G. Donsì, G. Ferrari, M. Poletto, Powder Technol. 191, 272-279 (2009)

[2] D. Barletta, G. Donsì, G. Ferrari, M. Poletto, P. Russo, AIChE J 53, 2240-2253 (2007)

[3] A. Castellanos, Adv. Phys. 54, 263 (2005)

[4] L. Konopka, J.J. Kosek, Electrostat. 87, 150 (2005)

[5] L. Bocquet, É. Charlaix, S. Ciliberto, J. Crassous, Nature 396, 735 (1998)

[6] A. Jarray, V. Magnanimo, S. Luding, Powder Technol. 341, 126-139 (2019)

[7] A. Gans, O. Pouliquen, M. Nicolas, Phys. Rev. E 101, 032904 (2020)

[8] W.A. Beverloo, H.A. Leniger, J. Van de Velde, Chem. Eng. Sci. 15, 260-269 (1961)

[9] A. Janda, I. Zuriguel, D. Maza, Phys. Rev. Lett. 108, 248001 (2012)

[10] M. Benyamine, P. Aussillous, B. Dalloz-Dubrujeaud, EPJ Web. Conf. 140, 03043 (2017)

[11] D. Gella, D. Maza, I. Zuriguel, Phys. Rev. E 95, 052904 (2017)

[12] I. Zuriguel, A. Garcimartín, D. Maza, L.A. Pugnaloni, J.M. Pastor, Phys. Rev. E 71, 051303 (2005)

[13] A. Janda, I. Zuriguel, A. Garcimartín, L.A. Pugnaloni, D. Maza, EPL-Europhys. Lett. 84, 44002 (2008)

[14] Z. Zou, P. Ruyer, P.Y. Lagrée, P. Aussillous, Phys. Rev. E 102, 052902 (2020)

[15] A. Abramian, L. Staron, P.Y. Lagrée, J Rheol. 64, 1227-1235 (2020) 